\title{
Performance studies of anisotropic flow with MPD at NICA
}

\author{
Petr Parfenov ${ }^{1,2, *}$, Arkadiy Taranenko ${ }^{1}$, Ilya Selyuzhenkov ${ }^{1,3}$, and Peter Senger ${ }^{1,3}$ \\ ${ }^{1}$ National Research Nuclear University MEPhI, Moscow, Russia \\ ${ }^{2}$ Institute for Nuclear Research of the Russian Academy of Sciences, Moscow, Russia \\ ${ }^{3}$ GSI Helmholtzzentrum für Schwerionenforschung, Darmstadt, Germany
}

\begin{abstract}
The Multi-Purpose Detector (MPD) at NICA collider has a substantial discovery potential concerning the exploration of the QCD phase diagram in the region of high net-baryon densities and moderate temperatures. The anisotropic transverse flow is one of the key observables to study the properties of dense matter created in heavy-ion collisions. The MPD performance for anisotropic flow measurements is studied with Monte-Carlo simulations of gold ions at NICA energies $\sqrt{s_{N N}}=4-11 \mathrm{GeV}$ using different heavy-ion event generators. Different combinations of the MPD detector subsystems are used to investigate the possible systematic biases in flow measurements, and to study effects of detector azimuthal non-uniformity. The resulting performance of the MPD for flow measurements is demonstrated for directed and elliptic flow of identified charged hadrons as a function of rapidity and transverse momentum in different centrality classes.
\end{abstract}

\section{Introduction}

Experimental and theoretical studies of the thermodynamical properties of quark-gluon matter are one of the top priorities worldwide in high-energy heavy-ion physics [1]. Transverse anisotropic flow measurements are one of the key methods to study the time evolution of the strongly interacting medium formed in nuclear collisions. In non-central collisions, the initial spatial anisotropy results in an azimuthally anisotropic emission of particles. The magnitude of the anisotropic flow can be defined via the Fourier coefficients $v_{n}\left\{\Psi_{m}\right\}$ of azimuthal distribution of the emitted particles with respect to the reaction plane [2]:

$$
\frac{d N}{d\left(\varphi-\Psi_{m}\right)}=\frac{1}{2 \pi}\left(1+2 \sum_{n=1}^{\infty} v_{n} \cos \left[n\left(\varphi-\Psi_{m}\right)\right]\right),
$$

where $\varphi$ - is the azimuthal angle of the particle, $n$ - is the harmonic order and $\Psi_{m}$ is the $m$-th order collision symmetry plane angle. $v_{1}$ and $v_{2}$ are called directed and elliptic flow, respectively.

\section{Simulation and analysis setup}

The main workflow is similar to the one described in the previous work [3, 4]. Ultrarelativistic Quantum Molecular Dynamics (UrQMD) [5, 6], and the Los Alamos QuarkGluon String Model (LAQGSM) [7, 8] were used to generate 4M and 100k events, respectively. The detector response was simulated using GEANT toolkit (version 3 and 4 - see in

\footnotetext{
*e-mail: terrylapard@gmail.com
} 
sec. 3.1). The resulting signals from the detector subsystems were used as input information for the reconstruction procedure.

The events were divided into classes of centrality ranging from 0 to $100 \%$ in steps of $5 \%$. In this work, the centrality determination is based on the multiplicity of the charged particles reconstructed by the Time-Projection Chamber (TPC), and the anisotropic flow analysis for $\mathrm{Au}+\mathrm{Au}$ collisions is presented for the two energies corresponding the highest and lowest ones of the NICA collider.

Tracks are selected according to the following criteria: $|\eta|<1.5 ; 0.2<p_{T}<2 \mathrm{GeV} / \mathrm{c}$; $N_{\text {hits }}^{T P C}>32 ; 2 \sigma$ DCA cut for primary particle selection; particle identification (PID) using $d E / d x$ from TPC and $m^{2}$ calculated from Time-of-Flight detector (TOF) [9]. Where DCA is the distance of the closest approach between the reconstructed vertex and a charged particle track.

\section{Results}

For the collective flow measurement, the event plane method was used [2]. The reaction plane was estimated from the energy deposition of the nuclear fragments in backward and forward rapidities in the forward hadron calorimeters (FHCal). $Q$-vector and event plane angle $\Psi_{m}^{E P}$ were calculated as follows:

$$
q_{x}^{m}=\frac{\sum E_{i} \cos m \varphi_{i}}{\sum E_{i}}, q_{y}^{m}=\frac{\sum E_{i} \sin m \varphi_{i}}{\sum E_{i}}, \Psi_{m}^{E P}=\mathrm{TMath}:: \operatorname{ATan} 2\left(q_{y}^{m}, q_{x}^{m}\right),
$$

where $E_{i}$ is the energy deposition in the $i$-th module of FHCal, and $\varphi_{i}$ its azimuthal angle. For $m=1$, the weights had opposite signs for backward and forward rapidities due to the antisymmetry of the $v_{1}$ as a function of rapidity. The anisotropic flow $v_{n}\left\{\Psi_{m}\right\}$ is calculated as follows:

$$
v_{n}\left\{\Psi_{m}\right\}=\frac{\left\langle\cos \left[n\left(\varphi-\Psi_{m}^{E P}\right)\right]\right\rangle}{R_{n}\left\{\Psi_{m}\right\}}, R_{n}\left\{\Psi_{m}\right\}=\left\langle\cos \left[n\left(\Psi_{m}^{E P}-\Psi_{m}\right)\right]\right\rangle,
$$

where $R_{n}\left\{\Psi_{m}\right\}$ is the event plane resolution, and $\Psi_{m}$ is the $m$-th order collision symmetry plane, which cannot be measured experimentally. To estimate the event plane resolution, the 2 -subevent method with extrapolation algorithm was used [2]. In this work $v_{1}$ and $v_{2}$ were measured with respect to the 1 -st order collision symmetry plane $\Psi_{1}$.

\subsection{Resolution correction factor}

In Fig. 1 the resolution correction factor for $v_{1}$ and $v_{2}$ is shown. The difference between LAQGSM and UrQMD is due to the fact, that the first model simulates nuclear fragments, while in the second one the spectators consist of neutrons and protons only. Hence, in the case of LAQGSM, more particles go through the beam hole in the center of FHCal detectors. Apart from that, the results show good performance in a wide centrality range from 0 to $80 \%$ for all energies.

In Fig. 2 the resolution correction factor for both flow harmonics using LAQGSM is presented. At $\sqrt{s_{N N}}=11 \mathrm{GeV}$, the energy deposition using GEANT4 increases compared to the values given from GEANT3 which can be a result of a more realistic hadronic shower simulation. 

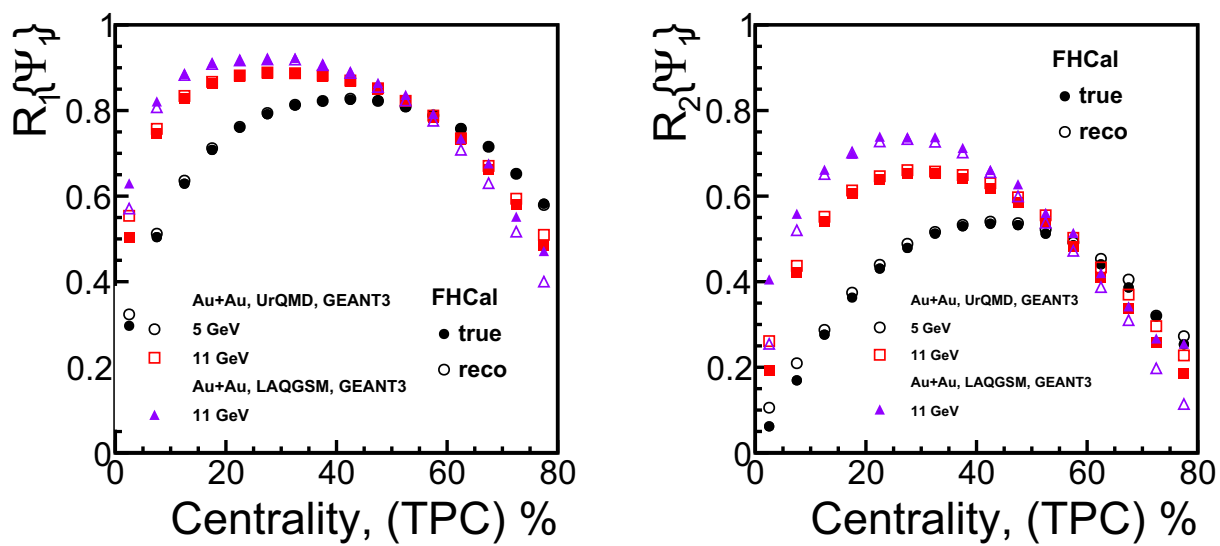

Figure 1. Resolution correction factor as a function of centrality for $v_{1}$ (left) and $v_{2}$ (right) for the UrQMD and LAQGSM event generators. The results from the GEANT3 simulation marked as true and one from the reconstruction procedure are marked as reco
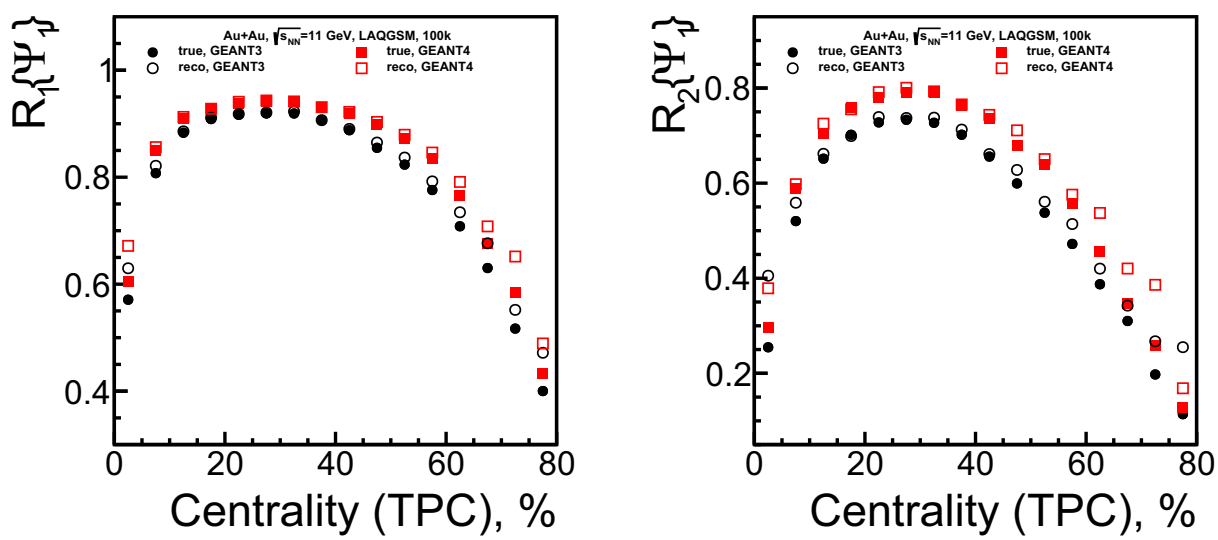

Figure 2. Resolution correction factor as a function of centrality for $v_{1}$ (left) and $v_{2}$ (right) for the LAQGSM event generator using GEANT3 (GEANT4) framework marked as black (red) dots

\subsection{Azimuthal anisotropic flow}

In Fig. 3 one can see the directed $v_{1}$ flow as a function of rapidity $y$. The plots in Fig. 4 depict the $p_{T}$-dependence of the elliptic $v_{2}$ flow. The results of the GEANT simulations (marked as "true") agree with the results from the reconstruction based on the detector response (marked as "reco"). The azimuthal flow is shown for the UrQMD event generator. Hadrons were identified via a combined PID method [9]. 

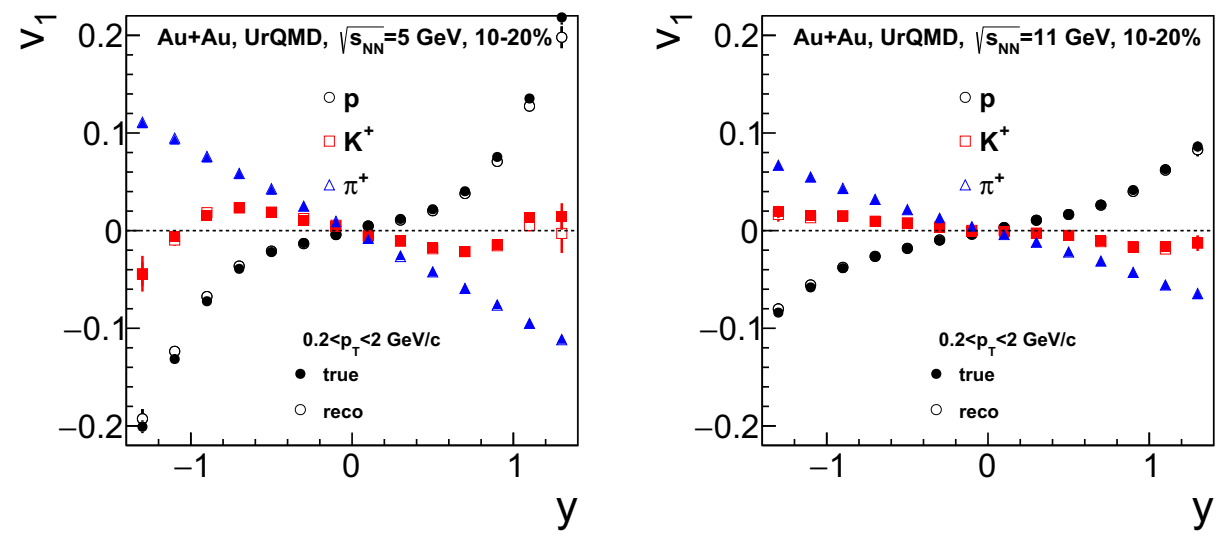

Figure 3. Directed flow $v_{1}$ as a function of rapidity $y$ for $\sqrt{s_{N N}}=5$ (left) and $11 \mathrm{GeV}$ (right). The results from the GEANT simulation are marked as true, and the ones from the reconstruction procedure are marked as reco
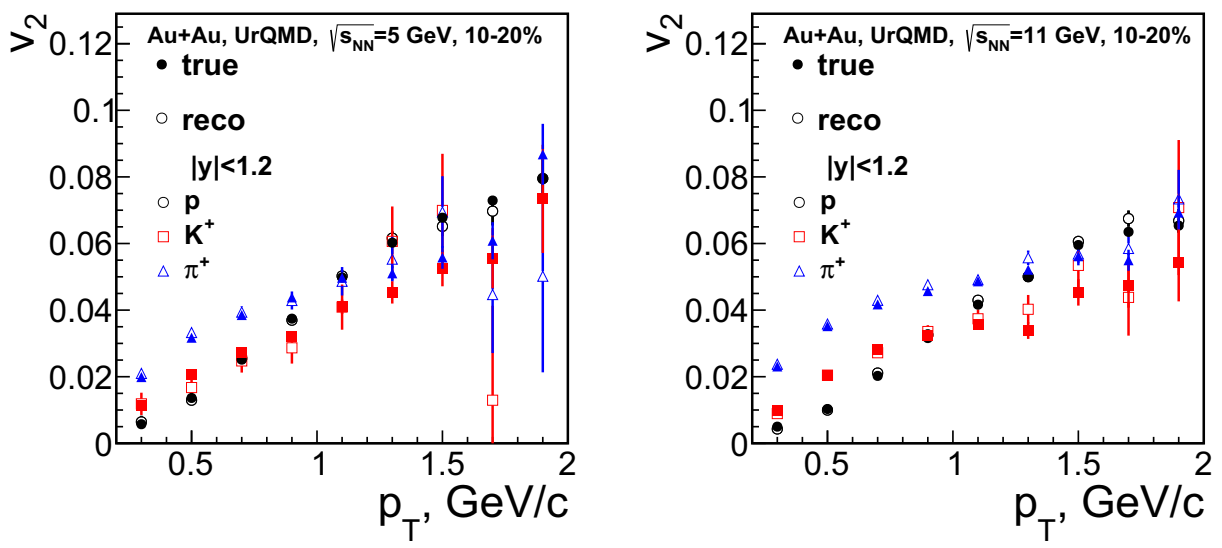

Figure 4. Elliptic flow $v_{1}$ as a function of transverse momentum $p_{T}$ for $\sqrt{s_{N N}}=5$ (left) and $11 \mathrm{GeV}$ (right). The results from the GEANT simulation are marked as true, and the ones from the reconstruction procedure are marked as reco

\section{Summary}

An anisotropic flow performance analysis with full reconstruction chain (which includes realistic particle identification) was shown for the MPD. The resolution correction factor increases using GEANT4 for the highest energy point available at NICA. The resolution correction factor as well as the directed and elliptic anisotropic flow simulated in GEANT are consistent with the reconstructed values which show a good performance of the MPD detector. 
Acknowledgments. The reported study was funded by RFBR according to the research project №18-02-00086 and the Ministry of Science and Education of the Russian Federation, grant №3.3380.2017/4.6.

\section{References}

[1] T. Czopowicz [NA61/SHINE Collaboration], Prog. Theor. Phys. Suppl. 193, 29 (2012). [arXiv:1201.5829 [hep-ex]]

[2] A. M. Poskanzer and S. A. Voloshin, Phys. Rev. C 58, 1671 (1998). [nucl-ex/9805001]

[3] P. Parfenov, I. Selyuzhenkov, A. Taranenko and A. Trutse, KnE Energ. Phys. 3, 352 (2018). doi:10.18502/ken.v3i1.1766. [arXiv:1712.09523 [hep-ex]]

[4] I. A. Svintsov, P. E. Parfenov, I. V. Selyuzhenkov and A. V. Taranenko, J. Phys. Conf. Ser. 798, no. 1, 012067 (2017)

[5] S. A. Bass et al., Prog. Part. Nucl. Phys. 41, 255 (1998) [Prog. Part. Nucl. Phys. 41, 225 (1998)]. doi:10.1016/S0146-6410(98)00058-1. [nucl-th/9803035]

[6] M. Bleicher et al., J. Phys. G 25, 1859 (1999). doi:10.1088/0954-3899/25/9/308. [hep$\mathrm{ph} / 9909407]$

[7] K. K. Gudima, S. G. Mashnik and A. J. Sierk, Los Alamos National Report LA-UR-016804 (2001). doi:10.1016/j.asr.2003.08.057. [nucl-th/0210065]

[8] S. G. Mashnik, K. K. Gudima, I. V. Moskalenko, R. E. Prael and A. J. Sierk, Adv. Space Res. 34, 1288 (2004). doi:10.1016/j.asr.2003.08.057. [nucl-th/0210065]

[9] A. Mudrokh and A. Zinchenko, EPJ Web Conf. 138, 11006 (2017). doi:10.1051/epjconf/201713811006 